\title{
ON THE INTENSITY DISTRIBUTION IN THE BANDS OF COMETARY SPECTRA
}

\author{
P. SWINGS AND M. NICOLET
}

\section{ABSTRACT}

The exceptional intensity distribution of bands of unsymmetrical molecules like $C N$ and $C H$ in a comet spectra can be explained by taking into consideration the following factors: $(a)$ the frequency of electronic absorption processes; $(b)$ the distribution in the vibrational levels (ground state) which is a function of the nuclear temperature $T_{\mathbf{r}}$ or of the equivalent radiation temperature $T_{\lambda} ;(c)$ the distribution in the rotational levels corresponding to the nuclear temperature. These factors are different functions of the heliocentric distance $r$.

I. Important progress has been made in recent years in our knowledge of the physical mechanisms which take place in the atmospheres of the comets; the most outstanding contributions in this field are those of $\mathrm{K}$. Wurm. ${ }^{\mathrm{I}}$ It seems useful to undertake a systematic rediscussion of the identifications of the emission bands observed in the spectra of comets; such a systematic work has been started in the department of astrophysics at Liége, Belgium. ${ }^{2}$

Actually, very great care must be taken in such identification work, as the intensity distributions and the structures of cometary bands differ widely from what we observe in normal laboratory spectra. This point has been advocated by Wurm on several occasions. In the course of our systematic discussions we had the feeling that there were still several points to be cleared up in connection with the prediction or discussion of the intensity distributions, as well as with the physical mechanisms present. The aim of this paper is to bring some precision into certain of these points. We shall restrict our considerations to the intensity distributions in rotational lines and vibrational bands of molecules which possess an electric dipole in their lowest electronic and vibrational state (such as $C N$ or $\mathrm{CH}$, but not $\mathrm{C}_{2}$ ); the fundamental difference between the behaviors of the two types of molecules has been discussed by Wurm.

I Cf. Zs.f. Ap., 15, Ir5, I938; Handbuch d. Ap., 7, 305, I936.

2 The first contribution of our department in this field has appeared recently; see M. Nicolet, "Les Bandes de $C H$ et la présence de l'hydrogène dans les comètes," Zs.f. Ap., 15, 154, 1938. Other papers by Nicolet are in preparation. 
Owing to the very low pressures prevailing in cometary atmospheres, the mean free path of the molecules is considerable. On the other hand, the excitation of the molecules has its primary origin in the solar radiation, the excitation by collisions being excluded. Under these conditions, what is the mechanism which prevails in the distribution of the molecules in the rotational levels of the lowest electronic and vibrational state?

2. Let $r$ be the distance, in astronomical units, from the comet to the sun. By the application of Stefan's law, and assuming $T_{\odot}=$ $5740^{\circ} \mathrm{K}$, it is found immediately ${ }^{3}$ that a body absorbing the solar radiation at a distance $r$ would take on a temperature $T_{\mathrm{I}} \sim 300 r^{-\mathrm{x} / 2}$. But this factor, $T_{\mathrm{I}}$, has no immediate meaning for the population in the discrete molecular levels in a gaseous atmosphere where collisions are practically absent.

3. We may, furthermore, try to find for different wave-length regions some kind of equivalent temperature $T_{\lambda}$ in complete similarity with the case of interstellar matter; 5 this equivalent temperature $T_{\lambda}$ must be such that the actual energy-intensity for wave-length $\lambda$ is equal to that of equilibrium radiation at temperature $T_{\lambda}$. This treatment seems at first logical in this problem, owing to the selective absorption between the different rotational levels. Assuming that the sun radiates like a black body, and using the appropriate dilution factor, we find for the distribution in the excited levels, at the distance $r=\mathrm{I}$ : for selective absorption at $\lambda \sim 4500 \mathrm{~A}$, the equivalent temperature $T_{\lambda} \sim 2000^{\circ} \mathrm{K}$; for selective absorption at $\lambda \sim$ I $\mu$, the equivalent temperature $T_{\lambda} \sim 800^{\circ}$; for selective absorption at $\lambda \sim$ Io $\mu$, the equivalent temperature $T_{\lambda} \sim 70^{\circ}$; for selective absorption at $\lambda \sim$ I00 $\mu$, the equivalent temperature $T_{\lambda} \sim 5^{\circ}$. In any case, since the pure rotational lines of the molecules with which we are concerned lie in the region from roo to roo० $\mu$ or more, we should find by application of Boltzmann's law

${ }^{3}$ See, for instance, A. Adel, V. M. Slipher, and R. Ladenburg, Ap. J., 86, 345, r937.

${ }^{4}$ For $T_{\odot}=6000^{\circ}, T_{\mathrm{r}}$ would be $3 \mathrm{r} 6 r^{-\mathrm{r} / 2}$, which means a difference of $\mathrm{I} 6^{\circ}$ at $r=\mathrm{r}$.

5 A. S. Edington, Internal Constitution of the Stars, 2 d ed., p. 37I ; for an application to the rotational levels of possible interstellar molecules, see P. Swings, M.N., 97, 21 2, I937. 
that all the molecules are practically in their lowest rotational level. This does not correspond with the observations.

4. Obviously, such a concentration in the lowest rotational level would be maintained only in the absence of perturbing processes. Collisions do not play any real role. But Wurm has called attention to an effect due to the electronic absorptions and emissions influencing the distributions in the rotational levels. He has shown that, owing to the differences in intensity of the $R$ and $P$ transitions in emission and absorption, higher rotational quantum numbers may be reached, as long as the frequency of the absorption processes in the photographic region is higher than $\mathrm{I} / \tau_{r}, \tau_{r}$ being the lifetime of the rotational levels.

This mechanism presumably takes place, but it is not easy to predict its actual effect. On the other hand, the $C N$ and $C H$ molecules show a very different distribution; the $C N$ molecules have their maximum population in the rotational levels near $K^{\prime \prime}=8($ at $r=\mathrm{I})$, whereas the $\mathrm{CH}$ molecules are concentrated in the two or three lowest levels. ${ }^{6}$ With Wurm's mechanism, the observed difference brings complications and probably implies quite different $\tau_{r}$ for $C N$ and $\mathrm{CH}$. We shall see later that, on the contrary, this difference appears conclusively when we assume another type of distributing mechanism.

5. It is possible to reach a result quite similar to that of Wurm by an alternative theory which enables an easy calculation of the effect in the general case; in fact, there are several other reasons which support our hypothesis and which will be developed in the following paragraphs.

The absorption of the solar radiation brings the cometary meteoric nucleus to a temperature $T_{\mathrm{x}}$ given by the application of Stefan's law, following section 2. In the absence of any perturbing process the rotational populations of the lowest electronic level of any molecule will be practically the equilibrium distribution ${ }^{7}$ cor-

${ }^{6}$ Following the identification by Nicolet, loc. cit.

7 As a first approximation we may neglect here the dilution factor; thus, the equivalent temperature due to the nucleus is the same for all wave lengths. On the other hand, it is obvious that the frequency of transitions between rotational levels in equilibrium at approximately $300^{\circ}$ is much higher than at $T_{\lambda} \sim 5^{\circ}$ or less. 
responding to $T_{\mathrm{I}}=300 r^{-\mathrm{I} / 2}$. The influence of the distance $r$ is thus apparent.

In certain cases Wurm's mechanism may complicate somewhat the distribution in the lowest levels; but we may state that a rotational distribution corresponding to $T_{\mathrm{x}}=30 \mathrm{cr}^{-1 / 2}$ seems sufficient for the interpretation of the observed features, as will be seen later. ${ }^{8}$

6. We shall immediately show how simply our assumption explains the different distributions of the $C N$ and $C H$ molecules. These molecules have very different moments of inertia:

$$
\begin{aligned}
& I^{\prime \prime}(C N)=\mathrm{I} 4 \cdot 10^{-40} \mathrm{gm} \mathrm{cm}^{2} \\
& I^{\prime \prime}(C H)=2 \cdot 10^{-40} \mathrm{gm} \mathrm{cm}^{2} .
\end{aligned}
$$

Introducing these figures into the expression of the rotational energy in the Boltzmann factor, we find that they explain immediately the difference in distribution.

7. The problem may be different in the case of the vibrational levels. Let us assume a frequency of vibration $\omega^{\prime \prime}=1000 \mathrm{~cm}^{-1}$. The first vibration-rotational bands will lie between Io $\mu$ and $\mathrm{I} \mu$; in this interval the equivalent temperature $T_{\lambda}$, following section 3 , ranges from $70^{\circ}$ to $800^{\circ}$. Thus, for the lowest levels the "nuclear" temperature $T_{\mathrm{r}}$ will have the predominant effect, but not for the higher-lying vibrational states.

8. Summarizing the preceding considerations, we may say that, excluding intermittent or exceptional phenomena, the intensity of a cometary band depends upon the following factors: (a) the frequency of electronic absorption processes, which depends upon the spectral region and the structure of the absorption bands; $(b)$ the distribution on the vibrational levels of the electronic ground state, which is a function either of the nuclear temperature $T_{\mathrm{I}}$ (for the low quantum numbers $v^{\prime \prime}$ ) or of the equivalent temperatures $T_{\lambda}$ (for high $\left.v^{\prime \prime}\right) ;(c)$ the distribution on the rotational levels corresponding to the nuclear temperature $T_{\mathrm{r}}=300 r^{-1 / 2}$. These factors are different functions of the distance $r$.

9. A verification of our hypothesis may be found in the good agreement obtained when we apply it to the identifications of $C N$

${ }^{8}$ An elementary calculation shows that the frequency of pure rotational transitions of molecules in equilibrium at $T_{I}=300^{\circ}$ is at least as high as the frequency of electronic absorptions near $\lambda 4500$ for an equilibrium at $T_{\lambda} \sim 2000^{\circ}$. 
or $C H$ lines or groups of lines. ${ }^{9}$ We shall not give here all the details; these will be published by one of us (M. Nicolet).

For any identification the resolving-power of the spectrograph used $^{\text {ro }}$ has to be considered first; the theoretical intensity of the rotational line must be examined next. Let $\Delta$ be the width in angstrom units of the monochromatic image of the slit on the plate. A convenient method for a first approach will be to represent each rotational line by a rectangle of width $\Delta$ and of height $I, I$ being the theoretical intensity of the line. $I$ is obtained immediately by classical formulae introducing the so-called intensity factor $i$ and the Boltzmann expression. If $\Delta$ is smaller than the wave-length separation between two lines, these will appear separated on the spectrogram; but in the opposite case, the intensities will be distorted and may be represented by the sum of the rectangles which are superposed. Thus, we may find a blackening on the spectrogram, either $(a)$ where the lines are very close together and not too weak (in the neighborhood of certain band heads) or (b) for the rotational quantum numbers giving maximum values of $I$. It may thus happen that a single branch will exhibit two marks on the plate: one near the head and one for the lines corresponding to the maximum values of $I$. For instance, the $P$ branch of the $(0,0)$ transition of $C N$, which has a head, may show two maxima; inversely the $R$ branch, which has no head, will exhibit only one maximum. In fact, this $R$ branch is blended with the $P$ branch of the (I, I) transition; thus, when the emissions starting from $v^{\prime}=\mathrm{I}$ are strong enough, this blending effect will complicate the aspect of the $R(\mathrm{o}, \mathrm{o})$ branch, although it is possible to interpret all the observed features. ${ }^{\text {II }}$

Io. The main results concerning $C N$ may be summarized as follows:

Let us take $\Delta=2 A$, which is approximately the case for the spectrograms of the Comet Brooks taken by W. H. Wright. ${ }^{12}$ As-

${ }^{9}$ We may safely say that many identifications, even of molecules like $C N$, are incomplete or erroneous!

Io We shall be concerned here exclusively with slit spectrograms.

Ir We want to emphasize the fact that the appearance of the spectrograms of the same comet obtained at the same moment with instruments of different resolvingpower may be very different. See W. H. Wright, Lick Obs. Bull., 7, 8, rgा 2 (Comet Brooks).

ז2 Ibid., n. Ir. Pl. No. $337: \Delta=$ r.9 A; Pl. No. $338: \Delta=2.4$ A. 
suming for $T_{\mathrm{I}}$, respectively, $280^{\circ}(r \sim \mathrm{I}, 2)$ and $420^{\circ}(\boldsymbol{r} \sim 0,5)$, we have drawn diagrams of the intensity distributions in the $(0,0)$ bands in two cases: case A: without considering the effect of the ( $I, I)$ transition; case $B$ : with consideration of the effect of the $P(\mathrm{I}, \mathrm{I})$ branch on $R(\mathrm{o}, \mathrm{o})$. These diagrams are shown in Figures I, 2, and 3 . The following results appear at once:

a) The band head ${ }^{\mathrm{x} 3}$ near $\lambda_{3} 88 \mathrm{I}$ has a rather sharp edge on the long wave-length side. Wurm has tentatively explained this phe-

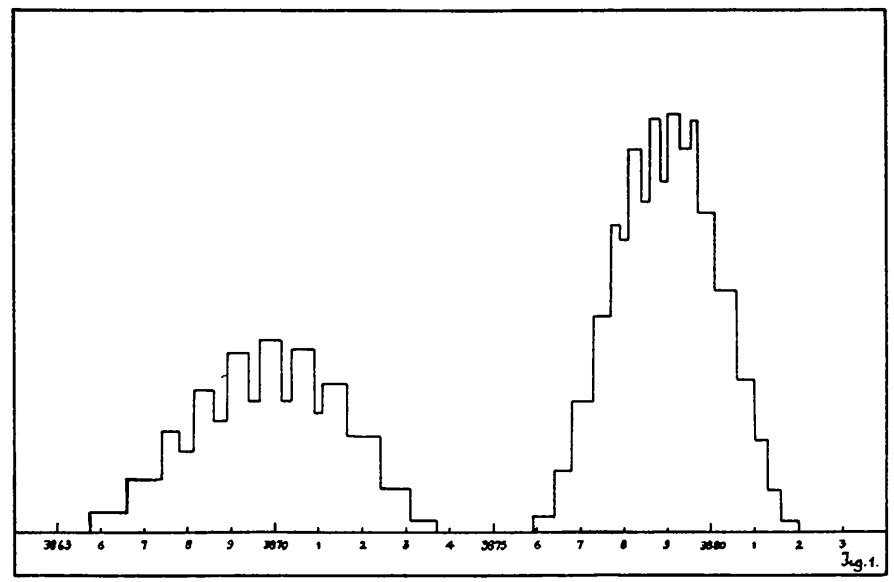

FIg. I. $-P$ and $R$ branches of the (o, o) transition in $C N . T_{\mathrm{I}}=280^{\circ} \mathrm{K}$. Without consideration of the $P(\mathrm{I}, \mathrm{I})$ band.

nomenon ${ }^{14}$ by considering the intensity of the solar radiation and the density of the solar absorption lines in the exciting wave-length region. The effect mentioned here gives an alternative explanation; it is particularly conspicuous at $T_{\mathrm{I}}=420^{\circ}$ (see Fig. 2).

b) Beside the head at $\lambda_{388} \mathrm{I}$, there is another maximum near $\lambda \lambda_{3878-3879 .{ }^{15}}$ The head will be prominent at small distances $r$ $\left(T_{\mathrm{r}} \sim 420^{\circ}\right)$; the opposite will happen at large distances $\left(T_{1} \ll 300^{\circ}\right)$.

c) The band near $\lambda_{3} 870$ is due to $R(0,0)$ in case A and to the blending of $R(\mathrm{O}, \mathrm{O})$ and $P(\mathrm{I}, \mathrm{I})$ in case B (Fig. 3). Case A corre-

I3 The laboratory head is at $\lambda_{3} 883.5$.

${ }^{14}$ Zs.f. Ap., 5, ro, 1932.

${ }^{15}$ If $\Delta<2$, this maximum may be separated from the band head; if $\Delta>2$, the separation will disappear and the microphotometer structure will be different, the principal feature being either the head or the maximum. 
sponds to great values of the distance $r$ (low population of the vibrational level $v^{\prime \prime}=\mathrm{I}$ ); Figure I gives us, then, a diffuse band with maximum at $\lambda \lambda$ 3869-3870. Case $B$ will happen for small distances $r$; the maximum of case $A$ at $\lambda 3870$ will then be distorted by another maximum of the $P$ (I, I) branch at $\lambda_{3} 868$.

d) Thus, at a very small distance $r$ we shall find simultaneously in-

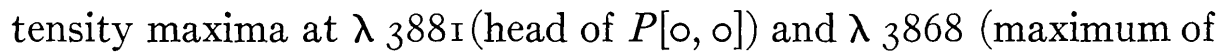

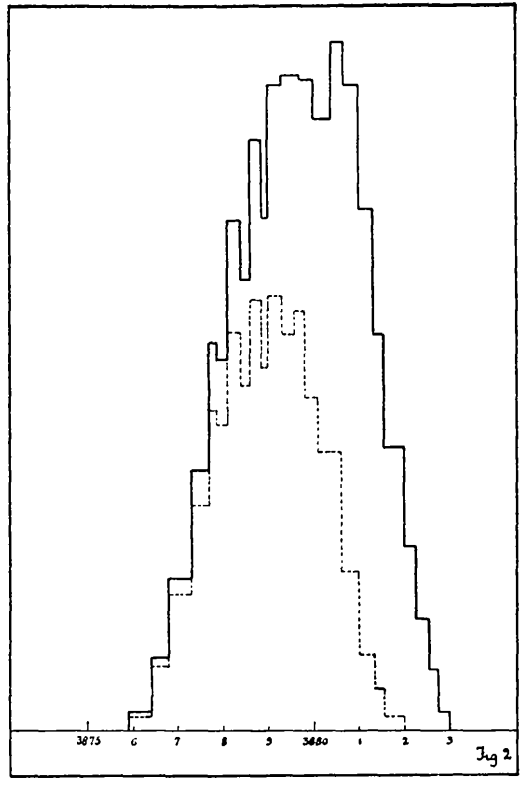

FIG. 2

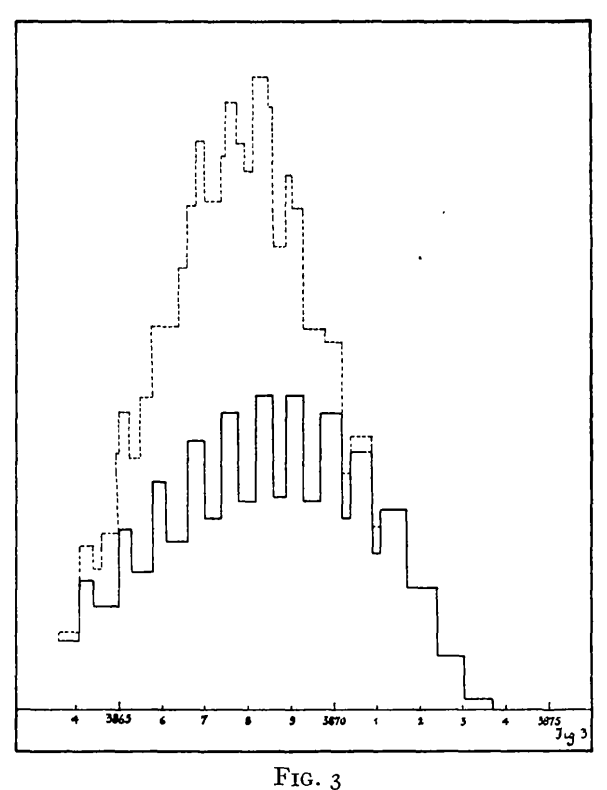

FIG. 3

FIG. 2.- $P$ branch of the $(0,0)$ transition in $C N$. Continuous curve: $T_{\mathrm{I}}=420^{\circ}$ $(r \sim 0.5)$; dotted curve: $T_{\mathrm{I}}=280^{\circ}(r \sim \mathrm{I} .2)$.

FIG. 3. $-R$ branch of the $(0,0)$ transition in $C N$. Continuous curve: $T_{\mathrm{I}}=420^{\circ}$ without blending effect of $P(\mathrm{I}, \mathrm{I})$; dotted curve: with consideration of $P(\mathrm{I}, \mathrm{I})$, assuming for convenience $I(\mathrm{I}, \mathrm{I})=\frac{1}{2} I(\mathrm{O}, \mathrm{O})(I=$ intensity).

$P[\mathrm{I}, \mathrm{I}])$; at a very large distance, intensity maxima will appear at $\lambda 3879$ (maximum of $P[\mathrm{o}, \mathrm{o}]$ ) and $\lambda \lambda 3_{3869-3870}$ (maximum of $R[\mathrm{o}, \mathrm{o}])$.

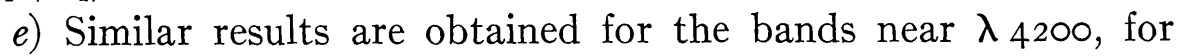
which our assumption brings clearly an intensity-distribution quite identical to the $(0,0)$ band. The band head near $\lambda_{42} I_{5}$ is especially conspicuous for $T \geqslant 420^{\circ}$, and the duplicity of the band at $\lambda$ 4I 96 is due to the superposition of $R(\mathrm{O}, \mathrm{I})$ and $P(\mathrm{I}, 2)$. 
f) An examination of the wave lengths obtained with slit spectrograms $^{16}$ discloses a satisfactory agreement with the preceding considerations; of course, we have to consider carefully the effects of the distance $r$. Similarly, there is no difficulty in explaining certain differences between the spectra of different comets.

g) The subdivision of certain $C N$ bands does not require special excitation processes of violent character; it is just a consequence of the preceding considerations.

h) Similarly, there is no need for two types of excitation, as suggested by A. Adel. ${ }^{17}$ For Comet Zlatinsky $(r \sim 0.7$ a.u.) the band at $\lambda_{3} 88 \mathrm{I}$.r corresponds, as has been stated by A. Adel and Wurm, to the wave lengths of the rotational lines $P$ (I3) or $P$ (I4) of the $(0,0)$ transition; but it is important to notice that actually the theoretically strongest line is $P(8)$ or $P(9)$; the observed effect is the result of the superposition of lines, owing to the value of $\Delta$. This appears conclusively in the diagrams, as well as in the behavior with varying distance $r$.

i) It is obvious that a Boltzmann distribution at temperature $T_{\mathrm{I}}$ is only a first, and possibly rather crude, approximation. The population in the lowest rotational level may be higher than in the case of a true Boltzmann distribution. If so, the lines observed near $\lambda \lambda 3864,3873$, and 4190 could be, respectively, the origins of the $(\mathrm{O}, \mathrm{O}),(\mathrm{I}, \mathrm{I})$, and $(\mathrm{I}, 2)$ transitions.

j) A careful investigation of the red system of $C N$ in comets is still lacking, the actual data by F. Baldet, ${ }^{18} \mathrm{~N}$. T. Bobrovnikoff, ${ }^{19}$ Dufay, Bloch, and Ellsworth, ${ }^{20} \mathrm{~J}$. Gauzit, ${ }^{2 \mathrm{x}}$ and R. Minkowski ${ }^{22}$ be-

${ }^{16}$ Comet Daniel: Campbell, Ap. J., 28, 229, r908; Comet Brooks: Wright, Lick Obs. Bull., loc. cit.; Comet Beliavsky: Bobrovnikoff, Pub. A.S.P., 43, 6I, I93I; Comet Zlatinsky: Slipher, Lowell Obs. Bull., 2, 67, Igr4; Comet Delavan: Curtiss and McLaughlin, Pub. U. Michigan, 3, 263, I923; Comet Mellish: Slipher, op. cit., p. I5I, r9ı6; Comet Finsler: Minkowski, Pub. A.S.P., 49, 276, r937; Comet Halley: Bobrovnikoff, $P$ ub. Lick Obs., 17, 443, I931.

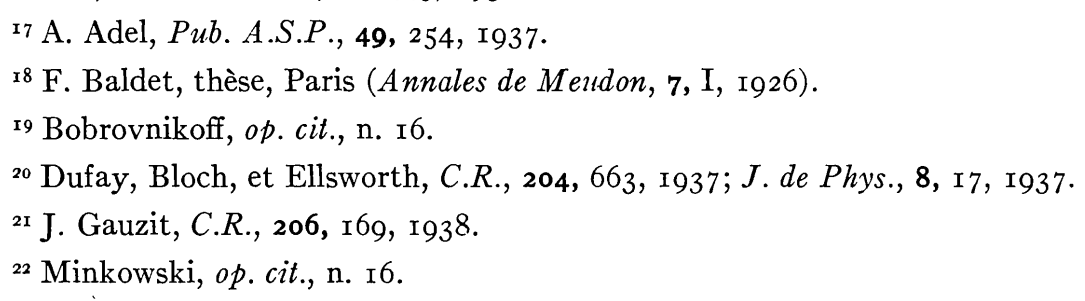


ing only preliminary in character. Owing to the different electronic transition $\left({ }^{2} \Pi \rightarrow^{2} \Sigma\right.$ instead of $\left.{ }^{2} \Sigma \rightarrow{ }^{2} \Sigma\right)$, the intensity distributions among vibrational bands, as well as the rotational lines, must be quite different.

I I. The identification of $C H$ by M. Nicolet ${ }^{23}$ was based on the same principle. Adopting $\Delta=2 A$ and $T_{\mathrm{r}}=300^{\circ}$, we obtain Figure 4 .

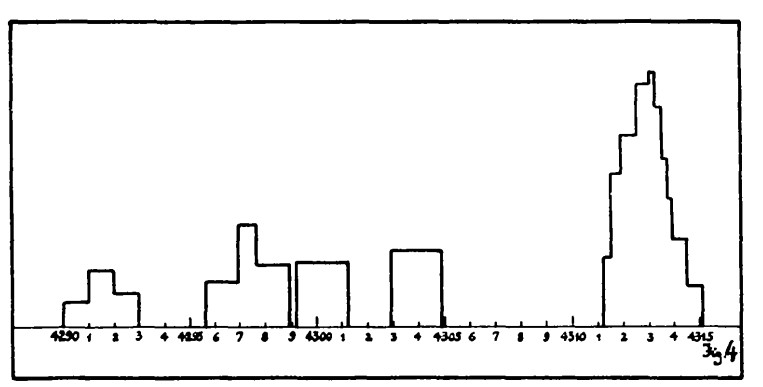

FIG. 4.- Intensity distribution in $C H$ between $\lambda 4290$ and $\lambda$ 43I 5 A. $T_{\mathrm{I}}=300^{\circ}$

This explains at once the following features: $(a) \lambda 43 \mathrm{I} 3$ is a rather narrow and strong emission; (b) $\lambda_{4297}$ and $\lambda$ 429r.5 are blends of two rotational lines; the intensity of $\lambda_{4297}$ is similar to that of the single line $\lambda 4304$; (c) when $\Delta>2 A$, a single band is observed from $\lambda_{4296}$ to $\lambda_{4301}$ (blend of three lines).

A discussion of the other electronic transition $B^{2} \Sigma \rightarrow \mathrm{X}^{2} \Pi$ of $C H$ will be published later by one of us (M. Nicolet).

UNIVERSITY OF LIÉGE

Department of Astrophysics April 1938

23 Nicolet, loc. cit. 